Ann. Biol. anim. Bioch. Biophys., 1978, 18 (1), 1-4.

\title{
Développement différentiel du squelette chez les bovins
}

par J. ROBELIN

Laboratoire de la Production de Viande, I.N.R.A.

Theix, Saint Genès Champanelle, 63110 Beaumont.

Summary. Differential development of the bovine skeleton.

Relative bone growth was studied on 220 cattle weighing 4 to $650 \mathrm{~kg}$ empty body weight. The skeleton allometric coefficient varied from 1.27 during fetal growth to 0.72 after birth. Significant differences in skeletal weight at the same empty body weight were found between breeds and between sexes. The allometric analysis of the weights of the different bones confirmed an increasing disto-proximal growth gradient ; the craniodistal growth gradient is discussed.

\section{Introduction.}

Les principaux travaux sur le développement différentiel du squelette chez les animaux domestiques ont surtout été effectués sur les ovins (Hammond, 1932) ou les porcins (Richmond et Berg, 1973 ; Davies, 1975). En effet, les résultats de Moulton et al. (1922) ou de Anon. (1966) concernent surtout le développement global du squelette chez les bovins, et non pas la croissance relative des différents os, et ceux de Seebeck (1973) ne couvrent qu'une courte période de la vie. Les résultats présentés dans cette étude décrivent l'évolution des différentes parties du squelette des bovins du stade fœtal $(4 \mathrm{~kg})$ à un stade voisin de 70 p. 100 de la taille adulte.

\section{Matériel et méthodes.}

L'ensemble des os de la demi-carcasse gauche de 220 bovins a été pesé après dissection complète. Sur 103 d'entre eux (dont 17 fœetus), les différents os de la demicarcasse ont été pesés séparément. Les animaux (mâles et femelles) utilisés, dont la répartition est illustrée sur la figure $1 \mathrm{~A}$ appartenaient à différentes races ou croisements (Charolaise, Frisonne, Limousine, Salers, croisement Charolais $\times$ Salers).

Les résultats ont été analysés selon la relation allométrique classique : $Y=a X^{b}$, dans laquelle b est le coefficient d'allométrie de $Y$ par rapport à $X$.

\section{Résultats.}

Evolution du poids du squelette en fonction du poids vif vide; variations selon la race ef le sexe des animaux. 
La croissance relative de l'ensemble du squelette par rapport au poids vif vide (fig. $1 \mathrm{~A})$ est significativement $(\mathrm{P}<0,01)$ plus élevée au cours de la vie foetale (coefficient d'allométrie $b=1,27)$, qu'après la naissance $(b=0,72)$. Ainsi, le squelette qui représente 9,0 p. 100 du poids vif vide chez un fœetus pesant $4 \mathrm{~kg}$, s'accroît jusqu'à 17 p. 100 du poids vif vide à $46 \mathrm{~kg}$, puis diminue régulièrement et atteint 8,2 p. 100 chez un bovin pesant $650 \mathrm{~kg}$.
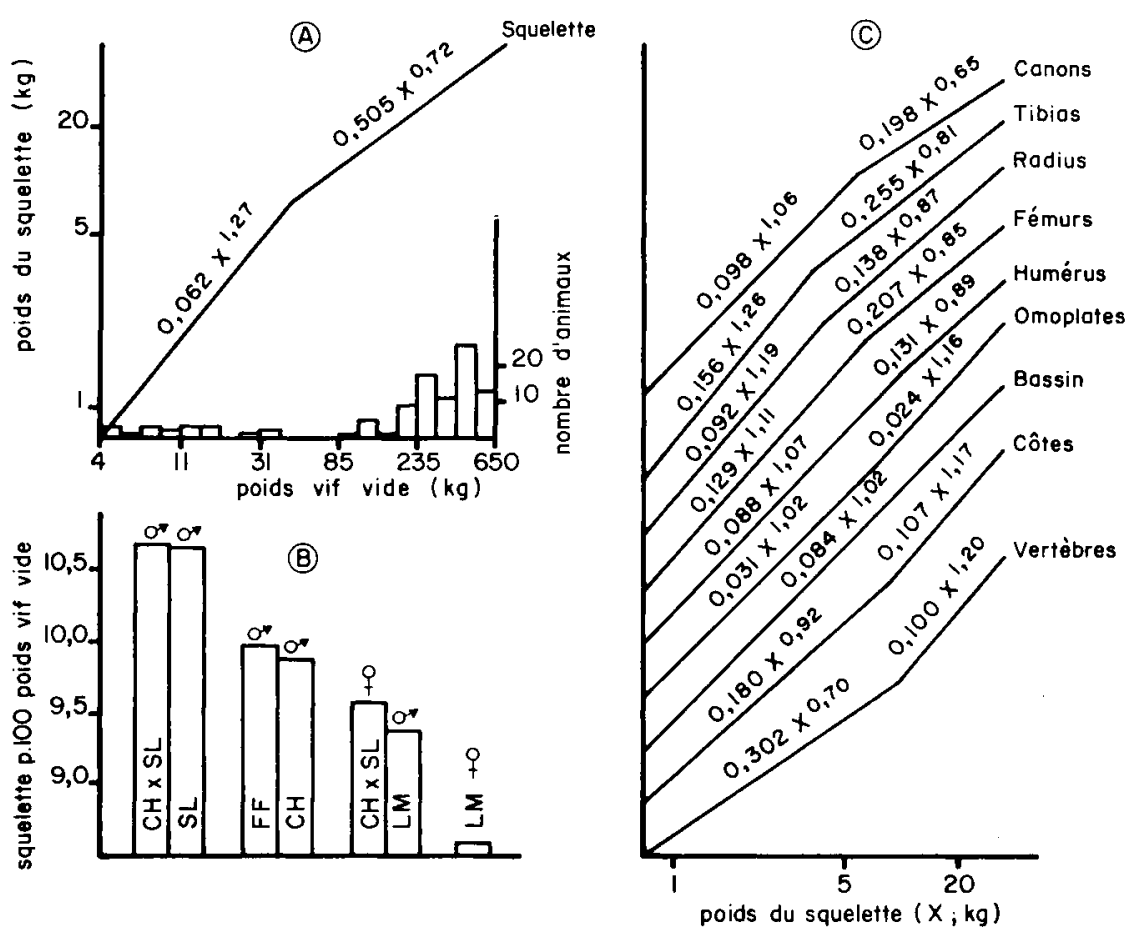

FIG. 1. - A : Evolution du poids du squelette chez les bovins en fonction du poids vif vide.

B : Variabilité selon la race ef le sexe.

CH : Charolais ; FF : Frison ; LM : Limousin ; SL : Salers.

$C:$ Développement différentiel des différentes parties du suqelette.

Il existe des différences significatives dans le poids du squelette (ramené au même poids vif vide de $455 \mathrm{~kg}$ ) entre animaux de races et de sexes différents (fig. $1 \mathrm{~B}$ ). Chez les animaux mâles issus de races rustiques ou de croisements avec une race rustique (Salers ef Charolais $\times$ Salers), le pourcentage de squelette dans le poids vif vide $(10,6$ p. 100) est significativement plus élevé que chez les taureaux de races spécialisées pour la production de viande ou de lait $(10,0$ chez les Charolais et les Frisons ; 9,4 p. 100 chez les Limousins). Enfin, le pourcentage de squelette est plus élevé chez les mâles que chez les femelles (9,4 contre 8,6 p. 100 dans la race Limousine ; 10,6 contre 9,6 p. 100 chez les bovins issus du croisement Charolais $\times$ Salers). 


\section{Croissance relative des différentes parties du squelette.}

L'analyse de l'évolution de la croissance relative des différents os du squelette (fig. $1 \mathrm{C}$ ) laisse apparaître trois ensembles homogènes, à savoir :

1. Les os du tronc, dont la croissance relative est significativement plus lente durant la phase fotale ( $b=0,70$ et 0,92 respectivement pour les vertèbres et les côtes) que pendant la phase postnatale $(b=1,20$ et 1,17 respectivement).

2. Les os des ceintures (omoplate et bassin) dont la croissance relative est isométrique (b voisin de 1) et reste pratiquement constante tout au long de la vie.

3. Les os des membres dont la croissance relative est très élevée chez les fotus (b compris entre 1,07 et 1,26) et beaucoup plus lente après la naissance (b compris entre 0,65 et 0,89 ), Enfin, les coefficients d'allométrie des os des membres postérieurs sont plus élevés que ceux des os des membres antérieurs au cours de la vie fœetale, alors que le phénomène est inversé après la naissance.

Ramenée à même poids de squelefte, la part représentée par les os des membres et significativement plus élevée chez les animaux de race Charolaise ou Frisonne (50,8 p. 100 du squelette total) que chez les taureaux Limousins (47,4 p. 100).

\section{Discussion.}

L'ensemble de ces résultats confirme une partie des phénomènes bien connus depuis les travaux de l'Ecole de Hammond sur les ovins, mais qui n'avaient pas, à notre connaissance, été quantifiés de façon précise sur les bovins, notamment au cours de la vie fœtale.

La croissance du squelette est certainement prioritaire chez le fœetus, ef l'évolution du coefficient d'allométrie par rapport au poids vif vide que nous avons observée $(1,27$ puis 0,72$)$ est comparable à celle qu'a obtenu Johnson (1974) également sur bovins. Cette priorité au cours de la vie fœtale va surtout aux os des membres, et plus particulièrement à ceux des parties distales, alors que le phénomène est inversé après la naissance. Ce dernier point rejoint ce qu'ont observé Seebeck (1973) sur des bovins, Paalson et Verges (1952) sur des ovins ou Davies (1975) sur des porcins dans la phase posinatale.

Dans le sens longitudinal, la hiérarchie de croissance ef son évolution sont beaucoup moins netfes. Bien qu'il ne soit pas discutable que la croissance des parties antérieures du squelette soit prioritaire chez le fœetus, il semble qu'après la naissance, il n'y ait pas simplement un déplacement continu vers l'arrière des zones de priorité. En effet, la croissance relative des os des membres antérieurs a été plus lente pendant la vie fotale el plus rapide après la naissance que celle des membres postérieurs. Paalson et Verges (1952) ont observé des résultats similaires sur des ovins âgés de 9 à 41 semaines. Enfin, Seebeck (1973) et Cuthberson et Pomeroy (1962) ont observé respectivement chez les bovins ef chez les porcins que la croissance relative des vertèbres cervicales était encore très rapide $(b>1,10)$ à un stade avancé du développement postnatal.

Les différences entre races dans le poids du squelette à même poids vif vide, ou dans sa répartition sont comparables en amplitude à celles qu'ont observées Mukhoty et Berg (1971) sur des bovins d'autres races. Bien qu'étant de moindre amplitude que 
les différences dans l'éłat d'engraissement, ces écarts dans la proportion de squelette contribuent également à établir des différences dans la valeur commerciale des animaux sur le plan de la production de viande.

Réunion Groupe Développement INRA/Productions animales Montpellier, 17-18 mai 1977.

\section{Références}

ANONYME, 1966. A comparison of the growth of different types of cattle for beef production. Rep. Major Beef Res. Proj., The Royal Smithfield Club, London.

CUTHBERSON A., POMEROY R. W., 1962. Quantitative anatomical studies of the composition of the pig at 50,68 and $92 \mathrm{~kg}$ carcass weight. II. Gross composition and skeletal composition. J. agric. Sci., 59, 215-223.

DAVIES A. S., 1975. A comparison of tissue development in Pietrain and Large white pigs from birth to $64 \mathrm{~kg}$ live weight. 3. Growth changes in bone distribution. Anim. Prod., 20, 45-49.

JOHNSON E. R., 1974. The growth of muscle, bone, fat and connective tissue in cattle from 150 day's gestation to 84 days old. Aust. J. agric. Res., 25, 1037-40.

HAMMOND J., 1932. Growth and development of mutton qualities in the sheep. Oliver and Boyd, Edinburg.

MOULTON C. R., TROWBRIDGE P. F., HAIGH L. D., 1922. Studies in animal nutrition. Il. Changes in proportions of carcass and offal on different plane of nutrition. Mo agr. expt. Station Res. Bull. $n^{\circ} 54$.

MUKHOTY H., BERG R. T., 1971. Influence of breed and sex on the allometric growth patterns of major bovine tissues. Anim. Prod., 13, 219-227.

PAALSSON H., VERGES J. B., 1952. Effects of plane of nutrition on growth and development of carcass quality in lambs. Part 1 : The effects of high and low planes of nutrition at different ages. J. agric. Sci., 42, 1-92.

RICHMOND R. J., BERG R. T., 1973. Bone growth and distribution in swine as influenced by liveweight, breed, sex and ration. Can. J. Anim. Sci., 52, 47-56.

SEEBECK R. M., 1973. The effect of body weight loss on the composition of Braham cross and Africander cross steers. II. Dissected components of the dressed carcass. J. agric. Sci., 80, 411 23. 